\title{
Antinuclear Antibodies in Nakajo-Nishimura Syndrome. A Bridge with Research on Refractory Autoimmune Diseases
}

\author{
Yutaka Inaba $^{1}$, Nobuo Kanazawa ${ }^{1}$, Kayo Kunimoto ${ }^{1}$, Fukumi Furukawa ${ }^{1,2^{*}}$ \\ ${ }^{1}$ Departments of Dermatology and Forensic Medicine, Wakayama Medical University, Wakayama, Japan \\ ${ }^{2}$ Takatsuki Red Cross Hospital, Osaka, Japan \\ Corresponding author: Fukumi Furukawa, Takatsuki Red Cross Hospital, 1-1-1 Abuno, Takatsuki, Osaka, Japan; \\ ffurukawa@takatsuki.jrc.or.jp
}

Short title: Antinuclear antibodies in Nakajo-Nishimura syndrome

\section{Keywords: Antinuclear Antibody, Nakajo-Nishimura Syndrome, Autoinflammatory Disease}

Nakajo-Nishimura syndrome (NNS) is an autosomal, recessively inherited disorder, which has been reported by Japanese physicians ${ }^{[1]}$. This disease is characterized by remittent fever, pernio-like skin rashes, nodular erythema-like skin eruptions and partial lipodystrophy. NNS is an immunoproteasome-associated autoinflammatory disorder caused by a mutation of the PSMB8 gene ${ }^{[2]}$. In general autoinflammatory diseases are not associated with autoantibody production because it is assumed that autoinflammatory disorders are caused by the dysfunction of innate immunity and/or the dysfunction of proteasomes that have been collectively designated as proteasome-associated autoinflammatory syndromes (PRAAS ${ }^{[3-5]}$. Autoinflammatory diseases were originally defined as diseases in which autoantibodies and autoreactive $\mathrm{T}$ cells were not detected, without activation of antigen-specific adaptive immune system, unlike autoimmune diseases ${ }^{[6]}$. However, in recent years, as we previously reported ${ }^{[7]}$, cases with the appearance of autoantibodies have been reported, and the boundaries are becoming vague.

We herein discuss the relationship between ANA and autoinflammatory NNS. We collected 9 cases with NNS, in which 5 cases (case number 1,3,4,8,9) showed positive ANA or anti-dsDNA antibody during the course ( Figure ).

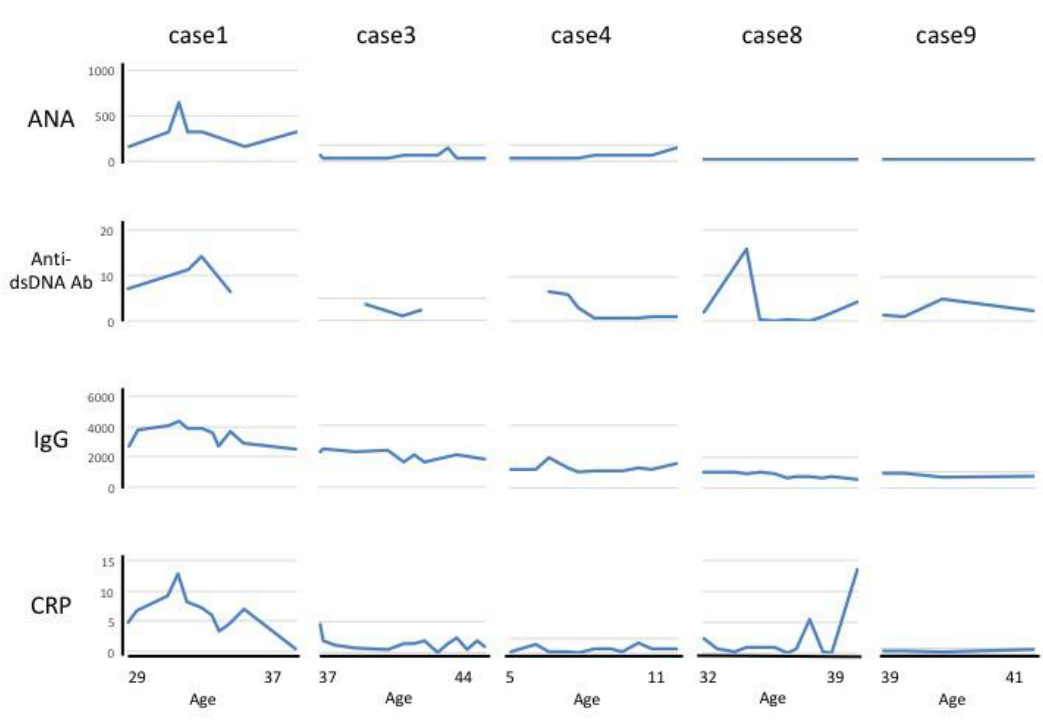

Figure; Changes in the values of serum antinuclear antibody, anti-dsDNA antibody, IgG, CRP over time in 5 subjects of Nakajo-Nishimura syndrome receiving continuous medical examination in Wakayama Medical University, Japan. 
In case $1^{[7]}$, ANA titer showed a transient rise sharply with CRP and IgG high values (although not shown in the figure, it reached 1280 times at this time) despite no significant changes of symptoms. In addition, ANA remained high although the decreasing trend of CRP after administration of a humanized anti-human IL-6 receptor monoclonal antibody. On the other hand, in case 8, the transient elevation of anti-dsDNA antibody was observed without elevations of CRP, IgG or ANA. Among them, other specific antibodies against extractable nuclear antigens etc. were not detected.

In recent years, the study of systemic lupus erythematosus (SLE) patients has revealed a central role for type I interferon (IFN) in disease pathogenesis ${ }^{[8]}$. In patients with SLE showing positive-ANA, serum IFN $\alpha$ is associated with the disease activity ${ }^{[9]}$, and IFN $\alpha$ contributed to the appearance of ANA in SLE patients with malignant carcinoid tumor $^{[10]}$. NNS also has aspects of IFN abnormality such as abnormally high serum IP-10, the involvement of both elevation of serum IFN $\alpha$ value (unpublished data) and activation of JAK-STAT pathway to IFN ${ }^{[11,12]}$.

Therefore, the autoantibodies in NNS is also expected due to abnormal production and response of IFN $\alpha$, but detailed pathological conditions need to be elucidated by accumulation and examination of further cases in the future. In other words, NNS will become a bridge with research on refractory autoimmune diseases.

\section{Conflict of interest}

We have no conflicts in this report. This was supported by grants from the Ministry of Health, Labor, and Welfare and the Japan Society for the Promotion of Science for N.K. (15K09780), K.K. (16K10171) and F.F. (17K09274).

\section{References}

1. Kanazawa N, Furukawa F: Autoinflammatory syndromes with a dermatological perspective. J Dermatol. 2007; 34(9): 601-618. doi:10.1111/j.1346-8138.2007.00342.x

2. Arima K, Kinoshita A, Mishima H, et al. Proteasome assembly defect due to a proteasome subunit beta type 8 (PSMB8) mutation causes the autoinflammatory disorder, Nakajo-Nishimura syndrome. Proc Natl Acad Sci USA. 2011; 108(36): 14914-14919. doi: 10.1073/pnas.1106015108.

3. Almeida De Jesus A, Raphaela Goldbach-Mansky R. Monogenic autoinflammatory diseases: concept and clinical manifestations. Clin Immunol. 2013; 147(3): 155-174. doi: 10.1016/j.clim.2013.03.016

4. McDermott MF, Aksentijevich I, Galon J, et al. Germline mutations in the extracellular domains of the $55 \mathrm{kDa}$ TNF receptor, TNFR1, define a family of dominantly inherited autoinflammatory syndromes. Cell. 1999; 97(1): 133-144. PMID: 10199409

5. Kunimoto K, Kimura A, Uede K, et al. A new infant case of Nakajo-Nishimura syndrome with a genetic mutation in the immunoproteasome subunit: an overlapping entity with JMP and CANDLE syndrome related to PSMB8 mutations. Dermatology. 2013; 227(1): 26-30. doi: 10.1159/000351323.

6. Furukawa F, Kanazawa N. Autoimmunity versus autoinflammation from the 2nd JSID-Asia-Oceania-Forum, Wakayama, Japan, 5th December, J Dermatol Sci. 2011; 63(2): 132-1327 doi: 10.1016/j.jdermsci.2011.05.001

7. Inaba Y, Kunimoto K, Kanasawa N, Furukawa F. Effects of a humanized anti-human IL-6 receptor monoclonal antibody on Nakajo-Nishimura syndrome. Trends Immunother. (in press) doi: http://dx.doi.org/10.24294/ti.v2.i3.1051

8. Pascual V, Farkas L, Banchereau J. Systemic lupus erythematosus: all roads lead to type I interferons. Curr Opin Immunol. 2006; 18(6): 676-682 doi:10.1016/j.coi.2006.09.014

9. Vallin H, Perers A, Alm GV, et al. Anti-double-stranded DNA antibodies and immunostimulatory plasmid DNA in combination mimic the endogenous IFN- inducer in systemic lupus erythematosus. J Immunol. 1999; 163(11): 6306-6313 PMID: 10570325

10. Ronnblom LE, Alm GV, Oberg KE. Possible induction of systemic lupus erythematosus by interferon- treatment in a patient with a malignant carcinoid tumor. J lntern Med. 1990; 227(3): 207-210 PMID: 1690258

11. Liu Y, Ramot Y, Torrelo A, et al. Mutations in proteasome subunit $\beta$ type 8 cause chronic atypical neutrophilic dermatosis with lipodystrophy and elevated temperature with evidence of genetic and phenotypic heterogeneity. Arthritis Rheum. 2012; 64 (3): 895-907 doi: 10.1002/art.33368

12. Inaba Y, Nakatani Y, Kunimoto K, et al. Enhanced phosphorylation of Janus kinase 1 in Nakajo-Nishimura syndrome. J Dermatol Sci 2017; 86 (2): e9 (abstract) doi: https://doi.org/10.1016/j.jdermsci.2017.02.026 